\title{
A Sphere Detection Based Adaptive MIMO Detection Algorithm for LTE-A System
}

\author{
Xuanli Wu ${ }^{1,2}$, Lukuan Sun ${ }^{1}$, Mingxin Luo ${ }^{1}$ \\ ${ }^{1}$ School of Electronics and Information Engineering Harbin Institute of Technology Harbin, China \\ ${ }^{2}$ Department of Electrical and Computer Engineering McGill University Montreal, Canada \\ Email: xlwu2002@hit.edu.cn,nic81@foxmail.com,822063769@qq.com
}

Received 2013

\begin{abstract}
An adaptive MIMO detection algorithm for LTE-A system which is based on sphere detection is proposed in this paper. The proposed algorithm uses M-algorithm for reference to remove unreliable constellation candidates before search, and the number of constellation reservation is adaptively adjusted according to SNR. Simulations of LTE-A downlink show that the BER performance of the proposed detection algorithm is nearly the same as maximum likelihood (ML) detection algorithm. However, the complexity is reduced by about $30 \%$ compared with full constellation sphere detection.
\end{abstract}

Keywords: Adaptive MIMO Detection; Sphere Detection; M-algorithm; LTE-A System

\section{Introduction}

As one of the core technologies employed in LTE and LTE-A system, MIMO (Multiple Input Multiple Output) technology can provide parallel channels in space, in which multiple data streams are transmitted. This obviously will improve the system transmission efficiently and obtain high spectrum efficiency under the condition of a certain bandwidth [1], which is unrealizable in SISO (Single Input Single Output) transmission. However, MIMO brings huge promotion in system capacity at the cost of enormous complexity in transceiver design, the biggest difference between MIMO and SISO receiver is that the received signals processed in MIMO receiver are with interferences between antennas. These interferences must be cancelled or reduced by special MIMO detection algorithms.

The design of a MIMO detection algorithms aims to find a trade-off between performance and complexity. The ML (Maximum Likelihood) algorithm can obtain the best detection performance in the sense of minimum BER [2], however, its complexity will increase exponenttially with the number of antennas and the modulation constellation. The linear detection algorithm, represented by ZF (Zero Forcing) and MMSE (Minimum Mean Square Error), has lower complexity, while the performance of such algorithms is still lower than ML algorithm. According to [3], the MMSE receiver is the baseline for performance evaluation of LTE system, and the UE could adopt more complex algorithms as technology evolves, such as QRM-MLD [4] or sphere detection [5]. Actually, QRM-MLD could be regarded as the evolution of sphere detection. It reserves $\mathrm{M}$ surviving symbol replica candidates in each stage to reduce the number of constellation searches, and the trade-off between performance and complexity is adjusted by the value of $\mathrm{M}$. Reference [6] proposed a threshold based adaptive control algorithm of surviving symbol replica candidates, and the control is applied to every stage. Reference [7] and [8] also put forward a threshold based adaptive candidate selection scheme, in which the noise is measured and evaluated in each stage. However, these algorithms are unsuitable for LTE system, because LTE supports double code words in space multiplexing and these two code words can employ different modulation and coding schemes, which will increase the complexity of threshold calculation. Furthermore, the threshold is related to an empirical constant in these algorithms, which is difficult to be determined in LTE system with various transmission schemes and complicated channel conditions.

Considering the characters of LTE system, a sphere detection based adaptive MIMO detection algorithm is proposed in this paper. The proposed detection drives from the QRM-MLD and reserves constellation candidates of each stage adaptively according to SNR. The adaptive detection can achieve the performance of ML detection with low complexity. What's more, this algorithm may utilize the feedback parameters and combine with CQI for the adaptive adjustment. 


\section{System Model}

The proposed detection is illustrated and simulated based on LTE-A downlink MIMO transmission. Figure 1 shows its general construction. Since employed OFDM, the MIMO detection is processed on each subcarrier. The MIMO transmission on each subcarrier with NT transmit antennas and NR receive antennas can be simplified in Figure 2.

On one subcarrier, the received signal vector

$$
\boldsymbol{Y}=\left[y_{1}, y_{2}, \cdots, y_{N_{\mathrm{R}}}\right]^{\mathrm{T}}
$$

is expressed as:

$$
\boldsymbol{Y}=\boldsymbol{H} \boldsymbol{X}+\boldsymbol{N}
$$

where, $\boldsymbol{H}=\left(h_{i j}\right)_{N_{\mathrm{R}} \times N_{\mathrm{T}}}$ is the channel impulse response, in which $h_{i j}$ denotes the fading characteristic from the $j$ th transmit antenna to the ith receive antenna. The transmitted signal vector on the identical subcarrier is denoted as $\boldsymbol{X}=\left[x_{1}, x_{2}, \cdots, x_{N_{\mathrm{T}}}\right]^{\mathrm{T}}$, and $\boldsymbol{N}=\left[n_{1}, n_{2}, \cdots, n_{N_{\mathrm{R}}}\right]^{\mathrm{T}}$ is the additive white Gaussian noise vector.

The transmission model in (1) is complex-valued, and the real-valued model can be written as

$$
\begin{aligned}
\tilde{\boldsymbol{Y}} & =\left[\begin{array}{c}
\operatorname{Re}(\boldsymbol{Y}) \\
\operatorname{Im}(\boldsymbol{Y})
\end{array}\right]=\tilde{\boldsymbol{H}} \tilde{\boldsymbol{X}}+\tilde{\boldsymbol{N}} \\
& =\left[\begin{array}{cc}
\operatorname{Re}(\boldsymbol{H}) & \operatorname{Im}(\boldsymbol{H}) \\
\operatorname{Im}(\boldsymbol{H}) & -\operatorname{Re}(\boldsymbol{H})
\end{array}\right] \times\left[\begin{array}{c}
\operatorname{Re}(\boldsymbol{X}) \\
\operatorname{Im}(\boldsymbol{X})
\end{array}\right]+\left[\begin{array}{c}
\operatorname{Re}(\boldsymbol{N}) \\
\operatorname{Im}(\boldsymbol{N})
\end{array}\right]
\end{aligned}
$$

where, $\tilde{\boldsymbol{Y}}, \tilde{\boldsymbol{H}}, \tilde{\boldsymbol{X}}$ and $N$ are all real-valued, and with double dimension as the corresponding complex-valued ones.

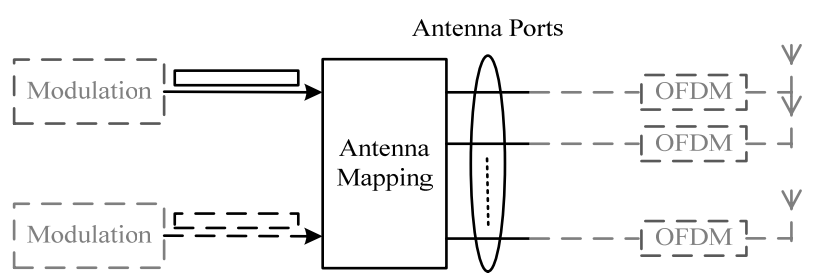

Figure 1. General construction of LTE-A downlink MIMO transmission.

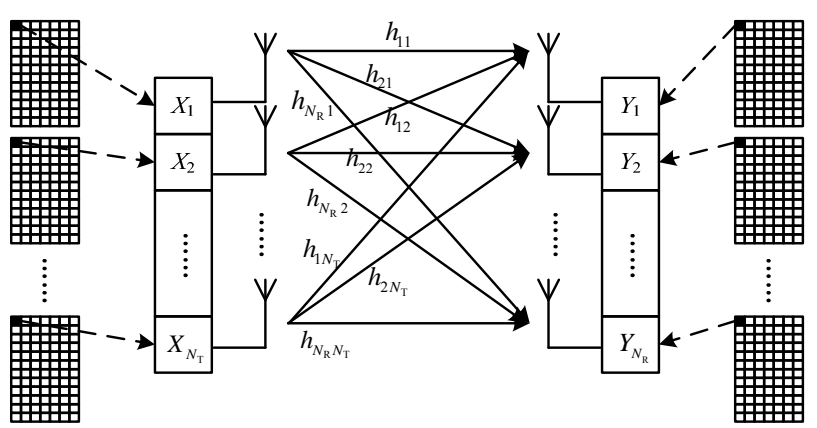

Figure 2. MIMO transmission on each subcarrier.

\section{Adaptive MIMO Detection}

\subsection{Conventional Sphere Detection}

The ML detection of the real-valued model in (2) can be written as

$$
\overline{\boldsymbol{X}}_{M L}=\arg \min _{\boldsymbol{X} \in \mathrm{S}}\|\tilde{\boldsymbol{Y}}-\tilde{\boldsymbol{H}} \tilde{\boldsymbol{X}}\|^{2}
$$

Let $c$ denote the known radius of a sphere with the center at $\tilde{Y}$ whose dimension is $N_{\mathrm{R}}$ (In this paper, the radius is set to be infinity to avoid missing the ML solution). Then the sphere detection can be expressed as

$$
\|\tilde{\boldsymbol{Y}}-\tilde{\boldsymbol{H}} \tilde{\boldsymbol{X}}\|^{2} \leq c^{2}
$$

When the QR decomposition is applied to $\tilde{\boldsymbol{H}}$, the real-valued channel matrix $\tilde{\boldsymbol{H}}$ can be expressed as

$$
\tilde{\boldsymbol{H}}=\tilde{\boldsymbol{Q}} \tilde{\boldsymbol{R}}=\left[\left(\boldsymbol{Q}_{1}\right)_{2 N_{\mathrm{R}} \times 2 N_{\mathrm{T}}}\left(\boldsymbol{Q}_{2}\right)_{2 N_{\mathrm{R}} \times 2\left(N_{\mathrm{R}}-N_{\mathrm{T}}\right)}\right]\left[\begin{array}{c}
\left(\boldsymbol{R}_{1}\right)_{2 N_{\mathrm{T}} \times 2 N_{\mathrm{T}}} \\
\mathbf{0}
\end{array}\right](5)
$$

where, $\tilde{\boldsymbol{Q}}$ is a $2 N_{\mathrm{R}} \times 2 N_{\mathrm{R}}$ unitary matrix, and $\tilde{\boldsymbol{R}}$ is a $2 N_{\mathrm{R}} \times 2 N_{\mathrm{T}}$ upper triangular matrix. Due to the norm preserving property of orthogonal matrix $\tilde{\boldsymbol{Q}}$, (4) could be written as

$$
\begin{aligned}
& \|\tilde{\boldsymbol{Y}}-\tilde{\boldsymbol{H}} \tilde{\boldsymbol{X}}\|^{2}=\left\|\tilde{\boldsymbol{Q}}^{\mathrm{H}} \tilde{\boldsymbol{Y}}-\tilde{\boldsymbol{Q}}^{\mathrm{H}} \tilde{\boldsymbol{H}} \tilde{\boldsymbol{X}}\right\|^{2} \\
& =\left\|\tilde{\boldsymbol{Q}}^{\mathrm{H}} \tilde{\boldsymbol{Y}}-\tilde{\boldsymbol{R}} \tilde{\boldsymbol{X}}\right\|^{2} \\
& =\left\|\left[\begin{array}{c}
\boldsymbol{Q}_{1}^{\mathrm{H}} \\
\boldsymbol{Q}_{2}^{\mathrm{H}}
\end{array}\right] \tilde{\boldsymbol{Y}}-\left[\begin{array}{c}
\boldsymbol{R}_{1} \\
\mathbf{0}
\end{array}\right] \tilde{\boldsymbol{X}}\right\|^{2} \leq c^{2}
\end{aligned}
$$

Hence, (7) can be obtained considering the matrix multiplication property

$$
\left\|\boldsymbol{Q}_{1}^{\mathrm{H}} \tilde{\boldsymbol{Y}}-\boldsymbol{R}_{1} \tilde{\boldsymbol{X}}\right\|^{2} \leq c^{2}-\left\|\boldsymbol{Q}_{2}^{\mathrm{H}} \tilde{\boldsymbol{Y}}\right\|^{2} \leq c^{2}
$$

Let $\boldsymbol{Y}^{\prime}=\boldsymbol{Q}_{1}^{\mathrm{H}} \tilde{\boldsymbol{Y}}$, then (8) can be obtain

$$
\left\|\boldsymbol{Y}^{\prime}-\boldsymbol{R}_{1} \tilde{\boldsymbol{X}}\right\|^{2}=\sum_{i=1}^{2 N_{\mathrm{T}}}\left(y_{i}^{\prime}-\sum_{j=1}^{2 N_{\mathrm{T}}} r_{i, j} \tilde{x}_{j}\right)^{2} \leq c^{2}
$$

Then, the VB (Viterbo Biglieri) algorithm [9], that employed iterative depth-first search strategy, can be adopted to find the ML solution.

\subsection{Preprocessing of MIMO Detection}

In sphere detection, how to find the point that is nearest to the vector $\tilde{X}$ is the main problem, and in order to find this point as soon as possible, some preprocessing is done before the MIMO detection.

SE (Schnorr Euchner) strategy [10] is an efficient way to make the search faster. The PEDs (Partial Euclidean Distance) of constellation candidates in each stage are sorted before search. Then the search is conducted in accordance with the order of increasing PEDs. From the 
perspective of geometry, SE strategy makes the search start from the nearest point to the center, so that it can reduce the radius with the quickest speed.

Actually, SE strategy makes the constellation candidates sorted in each stage. Similarly, the sorting of stages will make contribution to the search acceleration. It will increase the probability of finding nearer lattice point in early searches if sorting of stages is applied, that is the SQRD [11] (Sorted QR Decomposition). Figure 3 is the schematic diagram of sorting the stages.

Gram-Schmidt transform based SQRD and Householder transform based SQRD are two common algorithms. Reference [11] indicates that the unitary matrix $\boldsymbol{Q}$ and upper triangular matrix $\boldsymbol{R}$ generated from these two algorithms are different, and the matrix $\boldsymbol{Q}$ is not completely orthogonal, that is $\left\|\boldsymbol{Q}^{\mathrm{H}} \boldsymbol{Q}-\boldsymbol{I}\right\|^{2} \neq 0$. Gram-Schmidt transform based SQRD algorithm has lower complexity, while the matrix $\boldsymbol{Q}$ generated from Householder transform based SQRD algorithm will have better orthogonality. Therefore, Householder transform based SQRD algorithm is adopted in the preprocessing of MIMO detection.

\subsection{Adaptive MIMO Detection}

Conventional M-algorithm restricts the number of child nodes on each stage, i.e., the PEDs of all child nodes are calculated and $M$ child nodes with smaller PED are reserved while others are discarded. In order to avoid calculating and comparing PEDs of all child nodes, in the proposed algorithm, the constellation candidates of each stage are restricted before search. That is, the unreliable constellation candidates are removed to avoid unnecessary search.

According to the conclusion of SE strategy, the larger the PED of constellation candidate is, the more unreliable the constellation candidate is. Let $M$ denote the reserved constellation candidates of each stage, and each stage has the same value of $M$. Figure 4 takes 16QAM for example to illustrate the reservation of constellation candidates Normally, each father node has 4 child nodes, but in the proposed detection each father node will select $M$ more

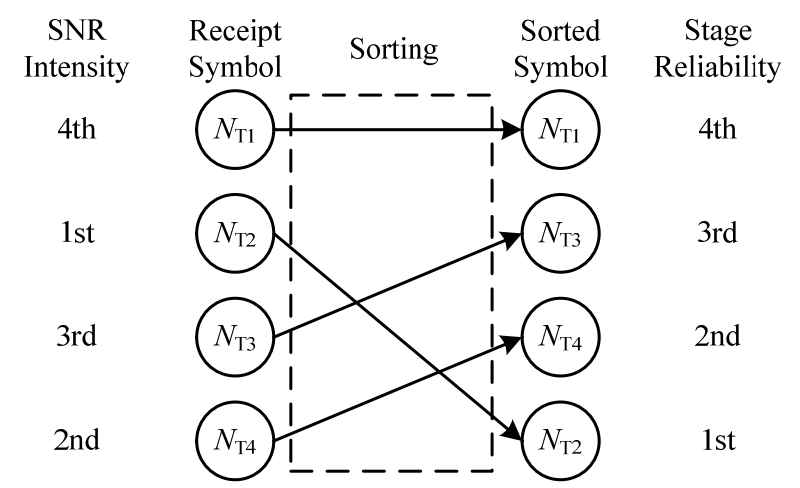

Figure 3. Sorting of stages.

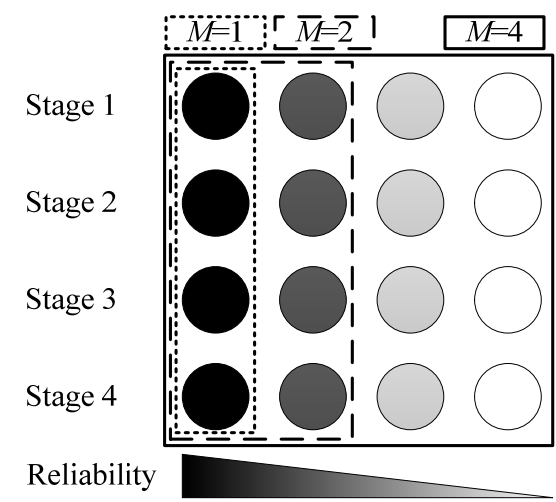

Figure 4. Reservation of 16QAM constellation candidates.

reliable constellation candidates as their child nodes, which contributes a drop of calculation in ML searches from $4^{N \mathrm{~T}}$ to $M^{N \mathrm{~T}}$ with $N_{\mathrm{T}}$ transmit antennas. When sphere detection is adopted, the number of search will be less. Hence, the value of $M$ determines the complexity of the algorithm.

Many simulations with different fixed values of $M$ show that the complexity of the algorithm is high in low SNR especially when $M$ is large or the modulation order is high, but starts to level off when SNR is greater than a certain value, and the BER performance difference between small value of $M$ and large value of $M$ is little in a certain range of SNR, but the complexity varies widely. Moreover, because there are only 2 constellation points in QPSK, the cancellation of constellation candidates will heavily decrease the BER performance. Therefore, the proposed detection is not for QPSK but for high-order modulation like 16QAM and 64QAM.

According to the analysis above, an adaptive MIMO detection is proposed. In this detection, SNR is divided into several intervals, and set different $M$ for different SNR interval. The detailed process of this algorithm is as follows:

1) Turn the received vector $Y$ and the estimated channel matrix $H$ into real-valued model according to (2).

2) Sort the constellation candidates of each stage from small PED to large PED by SE strategy.

3) Decompose $H$ into unitary matrix $Q$ and upper triangular matrix $R$ by Householder transform based SQRD.

4) Select appropriate value of $M$ according to current SNR.

5) Do the sphere detection, and obtain the estimation of real-valued transmit vector $\hat{\boldsymbol{X}}$.

6) Recover the complex-valued vector $X$ according to the order sorted by SQRD.

The adaptive adjustment scheme of $M$ could be regarded as a mapping between $M$ and SNR in a certain modulation scheme. This is similar to the mapping between CQI (Channel Quality Indicator) and SINR in LTE-A system. And the MCS (Modulation Coding Scheme) is 
determined by CQI. The interferences of LTE-A are mainly the adjacent cells with the same frequency, which can be taken as noise after interference randomization [12]. Hence, CQI is corresponding to MCS and SINR or SNR, while the modulation scheme and SNR define the value of $M$. That is to say UE can obtain the value of $M$ used for MIMO detection by CQI, which can be calculated through the reference signals sent by eNodeBs.

\section{Simulation Results}

The simulation is conducted mainly accord with the configurations of LTE-A Release 10 to realize real scenarios. However, in order to analyze the performance of the proposed MIMO detection algorithm preferably, procedures such as channel coding and scrambling were not involved. The urban macro model provided by ITU was adopted, and the ideal channel estimation was assumed. Table 1 gives the specific configuration of simulation parameters.

According to the simulations before, the adaptive adjustment scheme of $M$ is formulated in Table 2. Figure 5 and Figure 6 show the BER performance and complexity of the proposed detection algorithm in $2 \times 2$ and $4 \times 4$ MIMO system, respectively.

In Figure 5 and Figure 6, the performance of ML algorithm with full constellation candidates $(M=4)$ search is used to compare with the proposed detection algorithm. It can be found that the proposed adaptive detection algorithm almost achieves the same BER performance with ML algorithm using lower complexity. In $2 \times 2$ MIMO and $4 \times 4$ MIMO LTE-A systems, the average complexity is reduced by $27.61 \%$ and $32.27 \%$, respectively. In low SNR, the complexity reduction is more significant due to the small value of $M$.

Table 1. Configuration of simulation parameters.

\begin{tabular}{cc}
\hline Simulation Parameters & Configuration \\
\hline Bandwidth & $1.4 \mathrm{MHz}$ \\
Central Frequency & $2.5 \mathrm{GHz}$ \\
FFT Points & 128 \\
Frame Length & $1 \mathrm{~ms}$ \\
Length of CP & $4.69 \mu \mathrm{s}$ \\
Speed of UE & $1 \mathrm{~m} / \mathrm{s}$ \\
Modulation & $16 \mathrm{QAM}$ \\
\hline
\end{tabular}

Table 2. Adaptive adjustment scheme of $M$.

\begin{tabular}{cc}
\hline $\mathrm{SNR}(\mathrm{dB})$ & $M$ \\
\hline $\mathrm{SNR} \leq 7$ & 1 \\
$7<\mathrm{SNR} \leq 15$ & 2 \\
$15<\mathrm{SNR} \leq 20$ & 3 \\
$20<\mathrm{SNR}$ & 4 \\
\hline
\end{tabular}

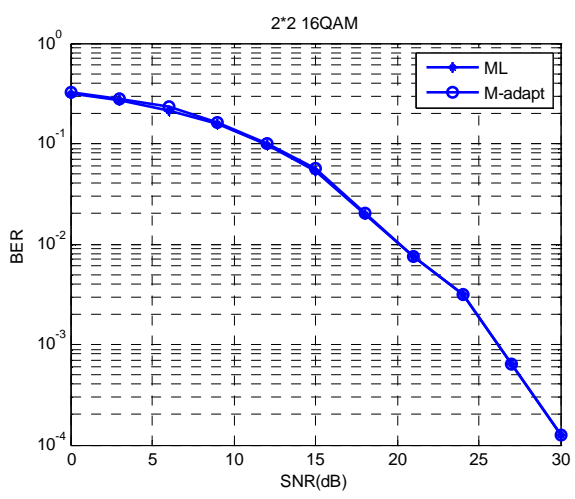

(a) BER performance of proposed detection algorithm

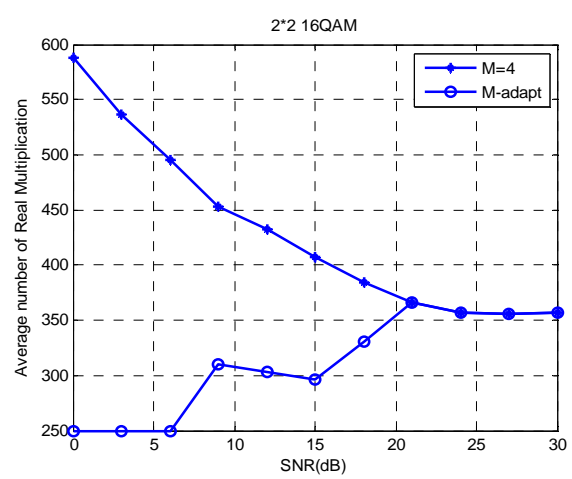

(b) Complexity of proposed detection algorithm

Figure 5. BER Performance and Complexity of 16QAM in 2 $\times 2$ MIMO System.

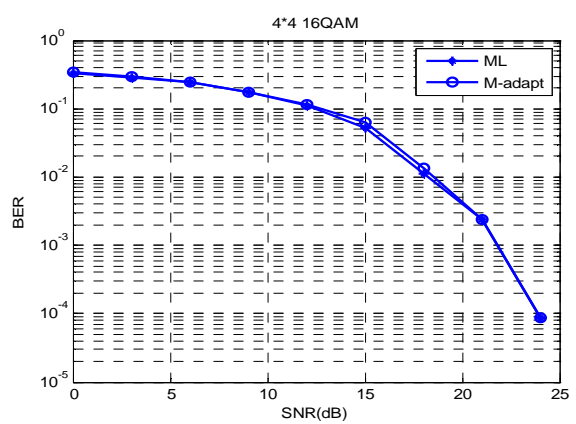

(a) BER performance of proposed detection algorithm

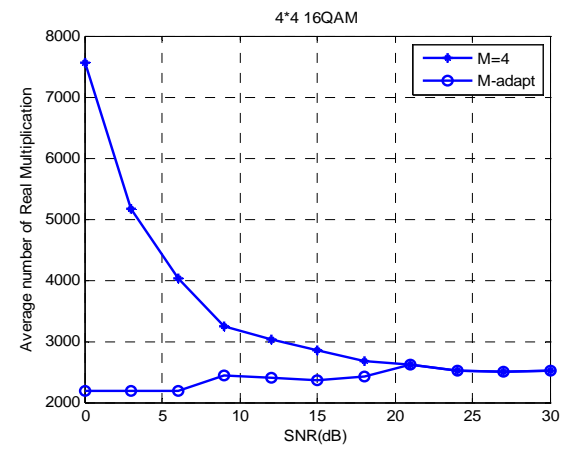

(b) Complexity of proposed detection algorithm

Figure 6. BER Performance and Complexity of 16QAM in 4 $\times 4$ MIMO System. 
From Table 2, it is obvious that $M$ is small when SNR is low, while in high SNR, $M$ is large. For each stage, there will be substantial differences in the reliability of constellation candidates after preprocessing with low SNR, so the candidates with lower reliability can be removed without anxiety. While in high SNR, the noise is so weak that every constellation candidate has much the same probability to become the ML solution, and the removal of candidates will decrease the possibility of finding ML solution, so the value of $M$ should be large in high SNR. Moreover, from the complexity figures of the proposed detection algorithm, it can be found easily that with the increase of $M$, the complexity increased accordingly, which due to the reason that the larger $M$ will increase total lattices in the receiver end.

\section{Conclusions}

This paper proposed a sphere detection based adaptive MIMO detection algorithm for LTE and LTE-A system. In this algorithm, the receiver can adjust the number of reserved constellation candidates of each stage according to current value of SNR. Simulation results show that the proposed algorithm almost achieves the same BER performance as ML detection algorithm, and compared with full constellation candidates sphere detection, in $2 \times 2$ MIMO and $4 \times 4$ MIMO LTE-A systems, the average complexity of the proposed algorithm is reduced by $27.61 \%$ and $32.27 \%$ respectively.

\section{Acknowledgements}

This work is supported by the National Basic Research Program of China (973 Program) under Grand No.2013 CB329003, Next Generation Wireless Mobile Communication Network of China under Grant No. 2012ZX 03001007-005, the Fundamental Research Funds for the Central Universities under Grant No. HIT.NSRIF2012020, Heilongjiang Postdoctoral Science-Research Foundation under Grant No. LBH-Q12081, and China Scholarship Council.

\section{REFERENCES}

[1] Y. K. Suk and H. Y. Kai, "Challenges in Migration to 4G Mobile Systems," IEEE Communications Magzine, Vol. 41, No. 12, 2003, pp. 54-59.

doi: $\underline{10.1109 / M C O M .2003 .1252799}$
[2] G. Forney, "Maximum Likelihood Sequence Estimation of Digital Sequences in the Presence of Inter-symbol Interference," IEEE Transations on Information Theory, Vol. 18, No. 3, 1972, pp. 363-378. doi: $\underline{10.1109 / \text { TIT.1972.1054829 }}$

[3] Q. H. Li, et al, "MIMO Techniques in WiMAX and LTE: A Feature Overview," IEEE Communication Magzine, Vol. 48, No. 5, 2010, pp. 86-92. doi: 10.1109/MCOM.2010.5458368

[4] K. Higuchi, H. Kawai, N. Maeda and M. Sawahashi, "Adaptive Selection of Surviving Symbol Replica Candidates Based on Maximum Reliability in QRM-MLD for OFCDM MIMO Multiplexing," IEEE Globecom, Dallas, 2004, Vol. 4, pp. 2480-2486.

[5] M. O. Damen, H. El Gamal and G. Caire, "On Maximum-Likelihood Detection and the Search for the Closest Lattice Point," IEEE Transactions on Information Theory, Vol. 49, No. 10, 2003, pp. 2389-2402. doi: 10.1109/TIT.2003.817444

[6] S. Nagayama and T. Hattori, "A Proposal of RM QRM-MLD with Independent Adaptive Control of Surviving Symbol Replica Candidates for MIMO-OFDM system," IEEE VTC 2007-Fall, Baltimore, 2007, pp. 452-456.

[7] W. Hou, T. Fujino and T. Kojima, "Adaptive Candidate Selection Scheme in QRM-MLD Algorithm for MIMO Detection," The 8th IEEE International Conference on Wireless and Mobile Computing, Networking and Communications, Barcelona, 2012, pp. 502-506.

[8] H. Kawai, K. Higuchi and N. Maeda, "Adaptive Control of Surviving Symbol Replica Candidates in QRM-MLD for OFDM MIMO Multiplexing," IEEE Journal on Selected Areas in Communications, Vol. 24, No. 6, 2006, pp. 1130-1140. doi: 10.1109/JSAC.2005.864027

[9] E. Viterbo and J. Boutros, "A Universal Lattice Code Decoder for Fading Channels," IEEE Transactions on Information Theory, Vol. 45, No. 5, 1999, pp. 1639-1642. doi: $\underline{10.1109 / 18.771234}$

[10] G. Zhan and P. Nilsson, "Reduced Complexity SchnorrEuchner Decoding Algorithms for MIMO Systems," IEEE Communications Letters, Vol. 8, No. 5, 2004, pp. 286-288. doi: 10.1109/LCOMM.2004.827376

[11] S. Aubert, F. Nouvel and A. Nafkha, "Complexity Gain of QR Decomposition Based Sphere Decoder in LTE receivers," IEEE VTC 2009-Fall, Anchorage, 2009, pp. 1-5.

[12] R. Bosisio and U. Spagnolini, "Interference Coordination Vs. Interference Randomization in Multicell 3GPP LTE System," IEEE Wireless Communications and Networking Conference, Las Vegas, 2008, pp. 824-829. 